\title{
Detection of Capacitor Switching Transients and L-G Fault Transients by Implementing Wavelet Transform
}

\author{
Prachi Thorat ${ }^{1}$, Satish Turkane ${ }^{2}$ \\ ${ }^{1,2}$ (E\&TC, Pravara rural engineering college, Pune university, India)
}

\begin{abstract}
Abstact: In a electrical power system, whenever the voltage goes below the desired level,there is no need to improve the voltage profile. This increasing voltage can be obtained by using capacitor bank/s, as switching ON a capacitor bank/s causes increase in voltage while switching OFF a capacitor bank/s causes decrease in voltage. But these switching action, if not specifically controlled give rise to transients which are undesirable and hazardous for the power system. This introduces a voltage disturbance detection approach [1] identifies voltage disturbance, and[2] discriminate the type of event which has resulted in the voltage disturbance, e.g. either a fault or a capacitor switching incident. Feasibility of the proposed disturbance detection approach is demonstrated based on digital time domain simulation of a distribution power system using the PSCAD software package. Finally by using wavelet transform discriminate the type of event on the basic of energy levels. The demand for educational and awareness programs are recommended in order to contribute and increase the use of online transaction in the sultanate.
\end{abstract}

Keyword: Capacitor switching,, distribution system, L-G,PSCAD wavelet transform, transient analysis.

\section{Introduction}

In the early days of development of the power system, electrical engineers were mainly concerned about 'keeping the lights ON'. They designed the power system to withstand outages. The main concern was to prevent the frequency of power system from deviating from $50 \mathrm{~Hz}$ after outages. With the development in technology, use of gadgets like computers, arc furnaces, photo copier machines etc. increased. Due to the use of these devices the load on the power system increases especially during the day time because of which many a times voltage level decreases and is needed to be increased. This task of increasing the voltage profile is done by switching $\mathrm{ON}$ of a capacitor bank in the power system. But this switching action gives rise to capacitor switching transients. L-G faults are most common of all the faults viz. L-L, L-L-G, L-L-L faults and introduces transients in the current and voltage waveforms. These transients are very hazardous to the power system as they cause severe problems to it and components present in it. For instance, at any moment it can crash the computer or data loss and other such problems. So it becomes necessary to know the cause of these transients so that they can be suppressed. With the advent of computers, it becomes easier to simulate a power system in various software's available for simulation. Today, various simulation software's like EMTP, ATP, PSCAD (Power System Computer Aided Design) are available in the market. Proposed study."THE IEEE 5 BUS SYSTEM $\square$ is considered and it is simulated in PSCAD. The data obtained from the simulation is exported to the Wavelet Toolbox of MATLAB (db4 is used for analysis) is for acquiring the various parameters like voltage and energy level of the waveform obtained in PSCAD.

\section{Proposed Disturbance Detection Scheme}

The proposed disturbance detection scheme (Fig.1) is composed of four main blocks; error signal generation, error signal analysis using wavelet, feature extraction, and decision making. Function of the error signal generation block is to extract the superimposed disturbances on the fundamental component of system voltages. This provides an error signal which is later analyzed using wavelet transform. Outputs of the wavelet transform are inputs to the feature extraction block which identifies specific signatures of the disturbances in the system. Finally, the decision making block discriminates various types of disturbances based on the error signal and the calculated features.

\section{1error signal generation}

The error signal generation block extracts the superimposed distortions on the measured voltages. The error signal is obtained by subtracting the fundamental component from the input signal. 


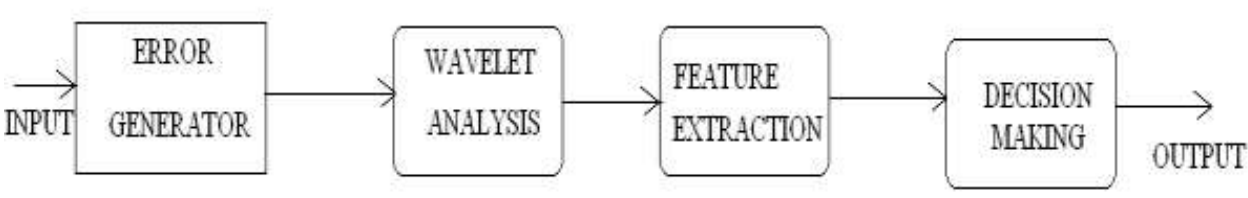

Fig.1 Proposed Disturbance Detection Scheme

\subsection{Wavelet Transform Analysis}

The wavelet analysis block transforms the error signal into different time-frequency scales. The wavelet transform provides information about the frequency content of a signal similar to the Fourier Transform (FT). However, contrary to the FT, wavelet transform is able to focus on short time intervals for high-frequency components and long intervals for low-frequency components, thus making it a well suited tool for analyzing high-frequency transients in the presence of low-frequency components. Wavelet transform is inherently more appropriate for non stationary and non periodic wide-band signals.

The wavelet transform of a continuous signal is:

$$
\mathrm{F}(\mathrm{a}, \mathrm{b})=\frac{1}{\sqrt{a}} \int_{-\infty}^{\infty} 1
$$

The values of wavelet coefficients $F(a, b)$ represent the projection of $f(t)$ along. Assume the center and width of function $\Psi(\mathrm{t})$ is zero and $\Delta \mathrm{t}$ in time domain, and zero and $\Delta \mathrm{w}$ in frequency domain. Then, the function is centered at $\mathrm{b}$ and has width of $\mathrm{a} \Delta \mathrm{t}$ in time domain and (1/a) $\Delta \mathrm{w}$ in frequency domain. To avoid generating redundant information, the base functions are generated discretely by selecting $\mathrm{a}=\mathrm{a} 0 \mathrm{~m}$ and $\mathrm{b}=\mathrm{nb} 0 \mathrm{a} 0 \mathrm{~m}$

Where, $\mathrm{a} 0$ and $\mathrm{b} 0$ are fixed constants with $\mathrm{a} 0>1$ and $\mathrm{b} 0>0, \mathrm{~m}, \mathrm{n} \varepsilon$, and is the set of integers. Setting a0and b0 to 2 and 1 respectively results in an orthonormal basis of $\mathrm{L}^{2}$ (IR) which is called dyadic- orthonormal wavelet transform. With this orthonormal basis, an algorithm of decomposing a signal into different time-frequency scales can be used which is called Multi resolution Signal Decomposition (MSD).

\subsection{Properties of Mother Wavelet}

Wavelets are families of functions generated form one single function, called as an analyzing wavelet or mother wavelet.

The mother wavelet must have the following properties

It must be oscillatory,

It must quickly decay to zero

It must have a zero average

It must be band pass

It must be integrate to

\subsection{Feature Extraction}

The purpose of feature extraction block is to identify specific signatures of the disturbances in the system. For example, a short circuit and a capacitor-switching incident result in disturbed voltages with different features. The wavelet transform breaks down the error signal into different time-frequency scales. Each scale represents the error signal in the corresponding band. The energy content of the scale signals relative to the error signal changes depending upon the type of disturbance. Therefore, the relative amplitudes of the scale signals with respect to the error signal are selected as the discriminating features.

\subsection{Decision Making}

A function of decision making block is to discriminate type of disturbances (L-G fault and Capacitor switching) as precisely as possible. The characteristic of each disturbance, example a fault, Capacitor switching, depends on several factors for example [1] type of event, e.g. single-phase-to-ground or phase-to-phase fault,[2] location of the event, [3] time instant of event and [4] network configuration.

In the decision making block, a probability functions is defined for the features and the decisions is made using the maximum linked (ML) criteria. This method is used here to discriminate various types of disturbances in a power system. 


\section{Method To Distinguish Between Capacitor Switching Transients And Other Transients}

One of the prime methods to distinguish between different types of transients i.e. Capacitor Switching and Fault transients is to use their energy content as the differentiating factor. We have to feed the data of transients into the wavelet toolbox of MATLAB and on the basis of energy content in 1 st and 2 nd Decomposition level, we can judge which type of switching action has caused the Transients. Energy content can be calculated by using the formula:

$$
\text { Energy }=\int \mathrm{IE}(\mathrm{t}) \mathrm{I} 2 \mathrm{dt}
$$

After the analysis in MATLAB the $\mathrm{d} 1$ and $\mathrm{d} 2$ for a LG fault is very hazardous as compared to that of Capacitor Switching Transients. Hence the energy threshold level of LG Fault for the given IEEE 5 BUS system is taken to distinguish between LG Fault Transients and Capacitor Switching Transients. Also the analysis for Back to Back Capacitor Switching and Isolated Capacitor Switching showed that the Energy threshold is higher for Back to Back Capacitor Switching. Hence this method can also be used to distinguish the Transients due to Back to Back Capacitor Switching and Isolated Capacitor Switching.

\section{FIGURES}

PSCAD work is now exported to the MATLAB $\square \mathrm{s}$ Wavelet toolbox. The analysis for IEEE 5 Bus System with LG Fault at Bus No 5, Back to Back Capacitor Switching at Bus No.5 and Isolated Capacitor Switching at Bus No 5 is done with sampling Frequency $10 \mathrm{KHz}$

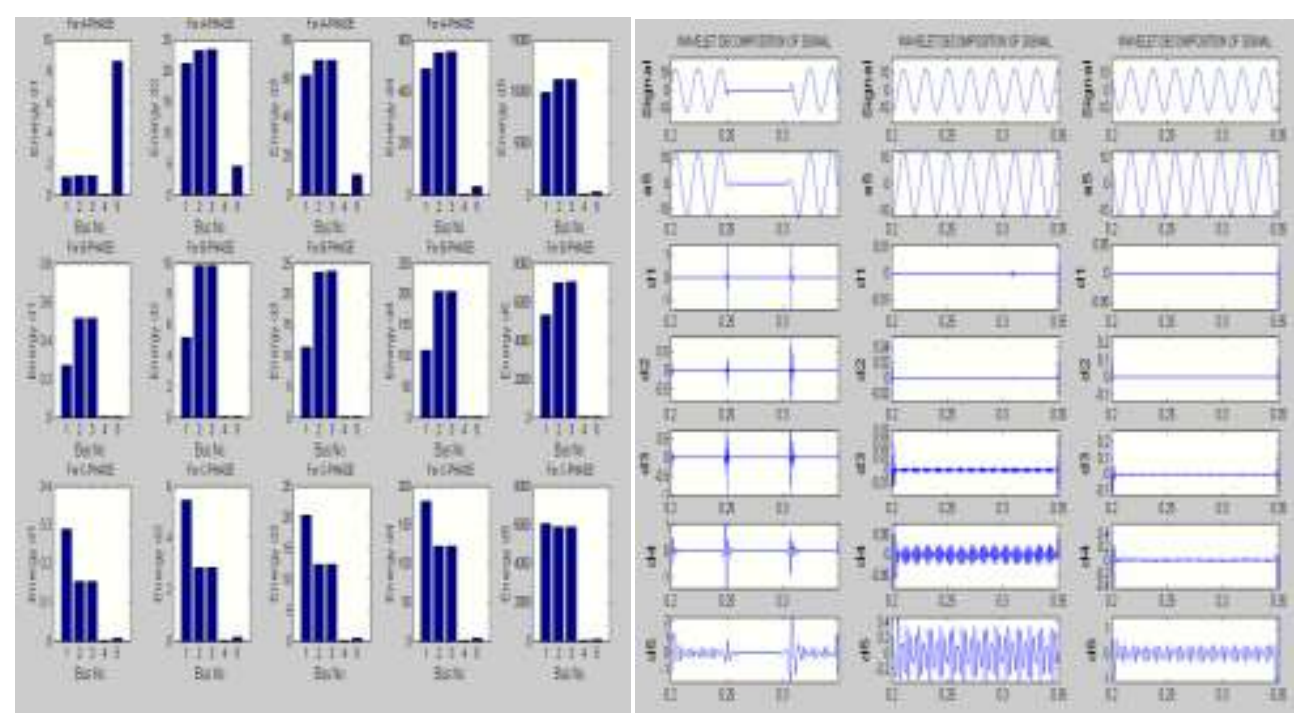

Fig.2 Energy Level at buses due to L-G fault

Fig. 3 Voltage at bus 5 due to L-G fault

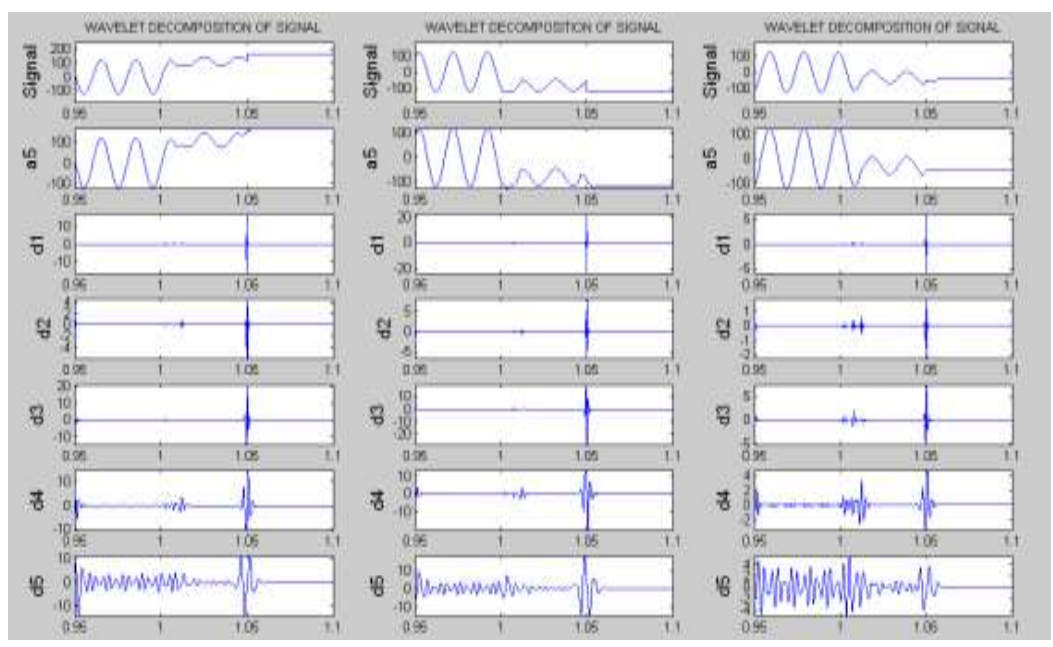

Fig. 4 Voltage at bus3due to back to back capacitor switching at bus 5 
Energy Content of Bus 5 with isolated Capacitor Switching is 0.0017 and $3.7723 \times 10-4$ for d1andd2 level decomposition while for Back to Back Capacitor Switching is 4.2706 and 2.6752.

Energy Content of Bus 5 with isolated Capacitor Switching is 0.0017 and 3.7723×10 -4 for d1andd2 level decomposition while for L-G Fault is 8.5568 and 4.5625.

\section{CONCLUSION}

The Proposed study introduces a voltage detection scheme for Power Quality applications. The scheme is based on Wavelet Transform. With the Simulation of IEEE 5 BUS system Capacitor Switching Circuit and LG Fault circuit in PSCAD and further analysis in MATLAB. Energy of the Transient produced due to Capacitor Switching depends on the magnitude of Capacitor being Switched ON along with the instant of Switching. Energy content of LG fault is more than that of a Capacitor Switching. Hence by analyzing the energy content of transients the cause of production of Transients can be predicted.

Presence of Capacitor in the network prohibits the Transients from entering into the system by providing them a path of less reactance through it. But this causes excessive heating of that Capacitor Bank and is one of the major reasons for frequent blowing up of the Capacitor Banks at the utilities.

This method (simply identifying Voltage Waveform) introduced new technique to identify the Transients due to capacitor switching and faults. The salient features of the new techniques are: Rapid Voltage disturbance detection Capability to identify the type of transient event which has caused voltage disturbance.

Performance of the new scheme is evaluated based on digital time-time domain simulation of a power distribution system, using the PSCAD.

\section{References}

[1] C. Sharmeela, M. R. Mohan, G. Uma and J. Baskaran., "A Novel Detection and Classification Algorithm for Power Quality Disturbances using Wavelet", Americian Journal of Applied Sciences, 3(10), pp-2049-2053,2006

[2] N.P. Subramaniam and K. Bhoopathy Bagan, “ Analysis of High Impedance Transients and Data Compression Using Wavelet Transform”, Serbian Journal of Electrical Engineering, Vol 3 ,No1, pp 19-31, June 2006.

[3] A.W. Galli and O. M. Nielsen, "Wavelet Analysis for Power System Transients", IEEE, Computer Applications in Power, pp 16 25 January 99.

[4] G. T. Heydt and A. W. Galli, "Transient Power Quality Problems Analyzed using Wavelets", IEEE Transactions on power delivery, vol 12, No 2, pp.908-915, april 97

[5] S. Santoso and P. Hofmann, "Power Quality assessment via wavelet transform analysis", IEEE Transactions on power delivery.vol 11, No 2, pp-924-930,april 96

[6] Masoud Karimi, Hossein Mokhtari and M. Reza Iravani," Wavelet Based on line Disturbance Detection for Power Quality Applications", IEEE Transactions on power delivery, vol 15, No 4, October 2000

[7] H.Y. Zhu and S. Chen, "Identification of Capacitor Switching Transients with Consideration of Uncertain System and Component Parameters" IEEE Transactions on power delivery, vol 23, No 1,pp-213 - 220,Jan 08.

[8] Lee, Wang and Huang, "A Literature Survey of Wavelets in Power Engineering Applications", Proc.Natl.Sci.Counc ROC(A),Vol24,No-4,pp 249-258,2000.

[9] W. Kanitpanyacharoean and S. Premudeep preechacharn, "Power Quality Problem Classification Using Wavelet Transformation and Artificial Neural Networks", IEEE, 2004.

[10] V. Matz, T. Radil, P. Ramos and A. C. Serra, "Automated Power Quality Monitoring System On-line Detection and Classification of Disturbances", Instrumentation and Measurement Technology Conferences, Warsaw Poland, May 1-3,2007. 